\title{
CURVAS ESFUERZO-DEFORMACIÓN AXIAL CALCULADAS PARA LAS ESPECIES DE BAMBÚ GUADUA ANGUSTIFOLIA KUNTH Y BAMBUSA OLDHAMII
}

AXIAL STRESS-STRAIN CURVES FOR TWO BAMBOO SPECIES
(GUADUA ANGUSTIFOLIA KUNTH AND BAMBUSA OLDHAMII)

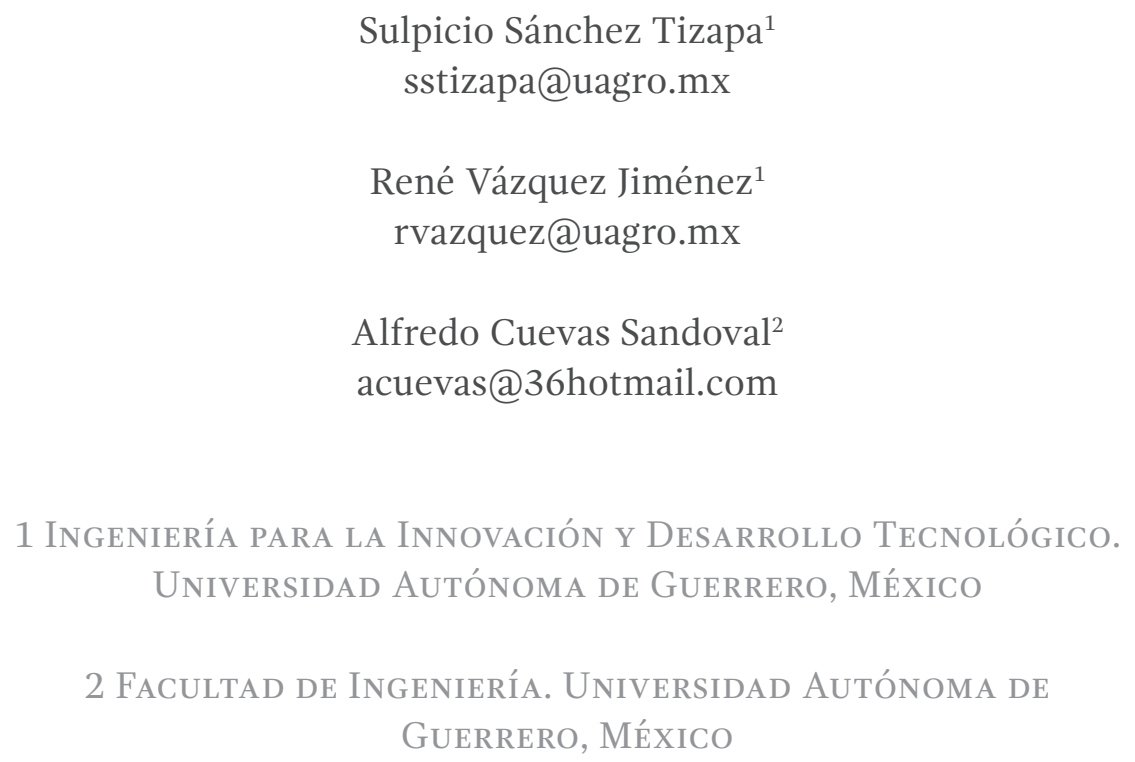


Para citar este artículo:

Sánchez-Tizapa, Sulpicio, Vázquez-Jiménez, René, Cuevas-Sandoval, Alfredo. (2019). Curvas esfuerzo-deformación axial calculadas para las especies de bambú Guadua Angustifolia Kunth y Bambusa Oldhamii. Espacio I+D, Innovación más Desarrollo. VIII(21), 97-117. doi: http://dx.doi.org/10.31644/IMASD.21.2019.a06

\section{RESUMEN}

Se presentan curvas de esfuerzo compresión-tensión contra deformación axial en dirección paralela a la fibra de pruebas realizadas en México para dos especies de bambú: Guadua Angustifolia Kunth y Bambusa Oldhamii. Las curvas de esfuerzo a compresión de la primera especie registradas en tres países (México, Ecuador y Colombia) muestran semejanza, sin importar las condiciones de ensaye, propiedades físicas-geométricas y posición del espécimen en la caña de bambú. Los valores máximos de esfuerzo de compresión- tensión se registraron en la especie Bambusa Oldhamii, 56.1 мра у 273.3 мра, respectivamente. Finalmente, con resultados registrados en México y Ecuador se obtuvieron las curvas compresión-tensión contra deformación axial de ambas especies. Las últimas gráficas muestran un mejor comportamiento del bambú respecto al concreto simple, por lo que el biomaterial podría utilizarse en la industria de la construcción.

\section{Palabras clave}

Bambú; Angustifolia; Oldhamii; curvas; tensión; compresión. 


\section{- Abstract-}

In this paper, experimental stress-strain curves of tensional and compressional tests carried out in Mexico for two bamboo species (Guadua Angustifolia Kunth and Bambusa Oldhamii) as wells as a comparison with those obtained in Ecuador and Colombia, are evaluated. For the Guadua Angustifolia Kunth, similar curve shapes for Mexican, Colombian and Ecuadorean bamboos were observed. This, in despite of variations in the test conditions, physic and geometric specimen properties, and original specimen position in the bamboo culm. Next, through a least square analysis, characteristic equations for these stress-strain curves were obtained. Bambusa Oldhamii species always shown the higher tensional and compressional strengths, 56.1 MPa and 273.3 MPa. Stress-strain curves for theses bamboo species were compared with that of unreinforced concrete. By considering the high strength and ductility capacity, the bamboo has a better behavior under axial stress than the unreinforced concrete and could be used for the building construction.

\section{Keywords}

Bamboo; Angustifolia; Oldhamii; Relationship; tension; compression. 
L os estados de Guerrero, Oaxaca y Chiapas tienen un alto peligro sísmico, coincidentemente es también la zona de menor desarrollo socialeconómico del país. Uno de los aspectos donde se refleja esta situación es la mala calidad de materiales e inadecuados procesos constructivos en las edificaciones. Esta combinación genera un elevado riesgo sísmico para la sociedad. Por esta razón es necesario proponer materiales de construcción ligeros, de bajo costo, resistentes y que puedan sustituir a los tradicionales como mampostería y concreto reforzado. Atendiendo a las características geográficas, físicas y meteorológicas de esta zona, un biomaterial que podría utilizarse es el bambú; el cual ha mostrado un comportamiento adecuado ante eventos sísmicos (Camacho \& Páez, 2002; González, 2006).

Un parámetro que permite definir un adecuado comportamiento sísmico es la relación resistencia a compresión entre peso específico de los materiales. Considerando valores medios, dicha relación es: a) $1042 \mathrm{~m}$ para concreto con resistencia a compresión, $f_{c}^{\prime}=25$ MPa (Gobierno de la CDMX, 2017); b) $3373 \mathrm{~m}$ en acero A-36 con esfuerzo de fluencia $f_{v}=248.2 \mathrm{MPa}$ (Instituto Mexicano de la Construcción en Acero, 2014); c) 8664 m para la especie Bambusa Oldhami. Adicionalmente, el bambú tiene una relación de esfuerzos últimos de tensión sobre compresión entre 2.8 y 4.9 (Álvarez, 2012), mientras que en el concreto simple no sobrepasa a 0.10 (Gonzáles y Robles, 2005). El bajo peso específico del bambú reduce las fuerzas sísmicas de diseño a valores del 13\% respecto a las obtenidas en sistemas tradicionales de mampostería (Kakkad \& Sanghvi, 2011). Referente a las propiedades mecánicas, el esfuerzo de tensión y módulo elástico están en los rangos 193 MPa - 340 MPa y 18 GPa-25.6 GPa, respectivamente según Dixon \& Gibson (2014). En la compresión, los esfuerzos van de 25.9 мPa a 33.5 MPa (Sánchez et al., 2016).

En el aspecto económico, considerando los daños del sismo $\mathrm{M}_{\mathrm{w}} 6.9$ ocurrido en Bhuj, India (1999), fue posible edificar construcciones con dobles muros de mampostería y refuerzo vertical de bambú en lugar del acero de refuerzo, las cuales tenían una relación de costo entre ambos materiales igual a 55\% (Sreemathi, 2002). En la ciudad de Chilpancingo, Guerrero, México, se construyó una casa de un nivel, donde el costo de los muros de bambú representó un 60\% del evaluado con muros de mampostería confinada (Ascencio, 2010).

En la República Mexicana existen pocas referencias técnicas sobre características y diseño del bambú. Por ejemplo, se estudiaron las propiedades mecánicas y físicas de tres especies que crecen en México con el interés de fomentar su uso en la construcción (Ordoñez-Candelaria \& Bárcenas-Pazos, 2014). También se reportan resultados en sistemas de cubiertas de marcos planos como alternativa en la construcción de vivienda económica; los marcos con armaduras de bambú y longitud de $6.0 \mathrm{~m}$ soportaron adecuadamente las cargas verticales y laterales generadas por efecto sísmico en una vivienda económica (Barragán-Trinidad et al., 2014). 
Por otro lado, la caracterización mecánica de materiales en especímenes pequeños ante diversas solicitaciones, tensión-compresión y esfuerzos flexionantes, es importante para extrapolar o definir el comportamiento de una estructura construida con estos materiales. De esta forma, por ejemplo, para la mampostería, se han propuesto leyes de comportamiento bilineal de muros a partir de ensayes de muretes (Sánchez et al., 2010).

\section{OBJETIVOS}

Los objetivos planteados son:

1. Evaluar las curvas esfuerzo axial contra deformación axial en una muestra pequeña de especies Guadua Angustifolia Kunth y Bambusa Oldhamii sembradas en Veracruz, México.

2. Obtener expresiones matemáticas de la relación esfuerzo normaldeformación normal de la especie Bambusa Oldhamii ensayada en México y de la Guadua Angustifolia Kunth ensayada en México, Colombia y Ecuador.

\section{MATERIALES Y MÉTODOS}

\section{III.1. Pruebas desarrolladas en México}

Los culmos de ambas especies ensayadas son originarios del estado de Veracruz, México y fueron previamente curados. Las pruebas realizadas en 16 especímenes a compresión paralela a la fibra se muestran en la Tabla 1, en la especie Guadua Angustifolia Kunth sólo se realizaron ensayes en especímenes con nodo.

\section{Tabla 1}

Especímenes ensayados a compresión

\begin{tabular}{ccc}
\hline Especie & Con nodo & Sin nodo \\
Bambusa Oldhamii & 4 & 6 \\
Guadua Angustifolia Kunth & 6 & - \\
\hline
\end{tabular}

La relación de esbeltez media (altura/diámetro exterior, $R E$ ) en los especímenes a compresión fue 1.9. Para medir la deformación normal se implementó el sistema mostrado en la Figura 1 y la deformación se registró a incrementos de $9.81 \mathrm{KN}$. También se realizaron pruebas de contenido de humedad y densidad. 


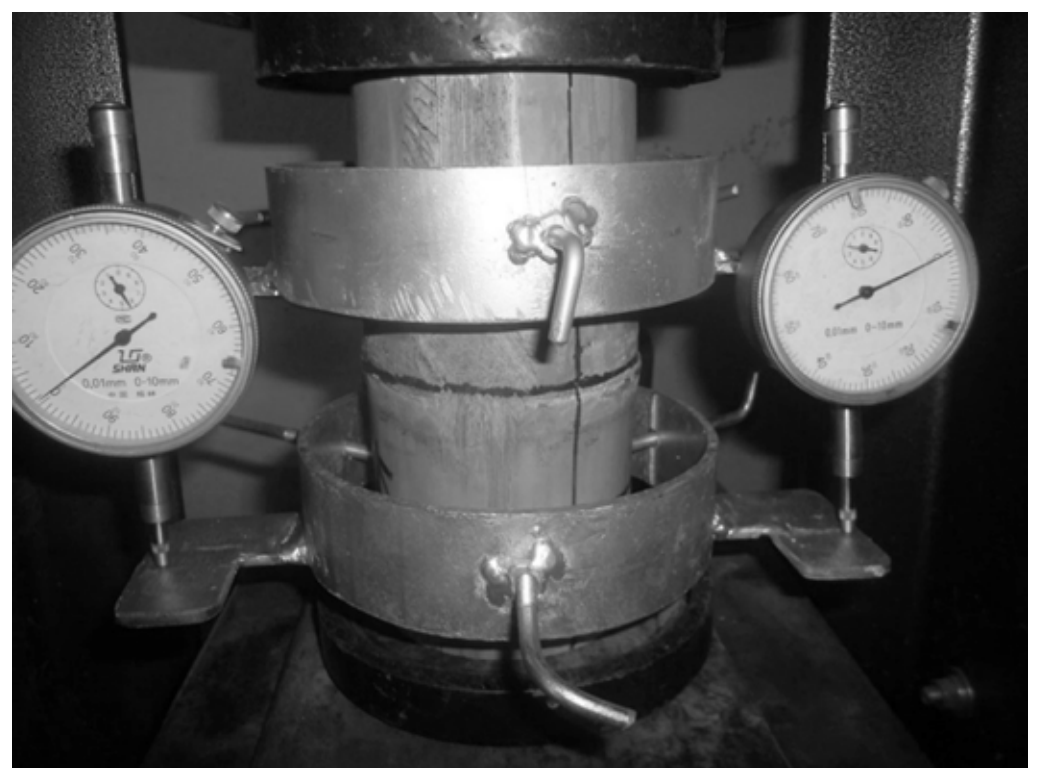

Figura 1. Sistema de medición de deformación en prueba de compresión

Respecto a las pruebas de tensión, Mendoza (2014) reporta resultados de 9 especímenes de la especia Bambusa Oldhamii, obtenidos de piezas con $0.90 \mathrm{~m}$ de longitud. La Figura 2 muestra la geometría y forma final de las muestras.A lo largo de la historia en México, varios han sido los programas que se han impulsado con el objetivo de fomentar la lectura.
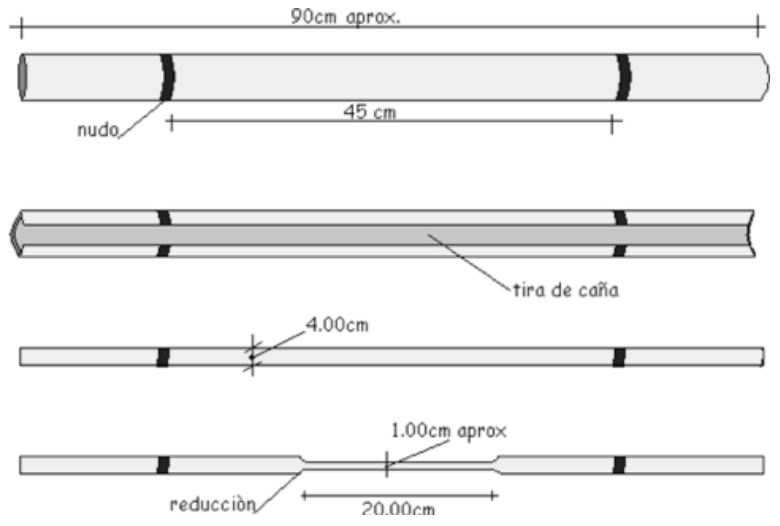

a)

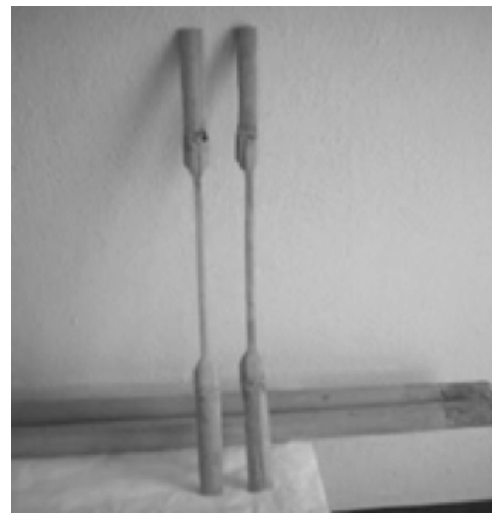

b)

Figura 2. a) Extracción y geometría, b) Muestra de especímenes a tensión. (Mendoza, 2014)

Por otro lado, no existen normas mexicanas para el ensaye de especímenes de bambú, por lo que en este trabajo se utilizó sólo como guía la norma iso 22157-1: 2004 (International Organization for Standardization, 2004). Sin embargo, la instrumentación de los especímenes a compresión se realizó con deformímetros mecánicos siguiendo el procedimiento de ensaye realizado en cilindros de concreto a compresión a diferencia de la utilización de 
galgas extensométricas propuestas en la norma Iso; en Colombia también se instrumentó con deformímetros mecánicos (González, 2006). Respecto a la prueba de tensión, la medición de la elongación se realizó con un transductor de desplazamiento lineal (LDVT) adherido a la probeta (Mendoza, 2014).

\section{III.2. Datos obtenidos en Ecuador}

En Ecuador, un extenso trabajo experimental fue desarrollado para determinar las propiedades mecánicas y estructurales de la especie Guadua Angustifolia Kunth, con el objetivo de proponer valores para diseñar construcciones de forma segura. La información utilizada en este documento corresponde a 11 curvas esfuerzo-deformación registradas en pruebas de compresión paralela a la fibra en especímenes con relación de esbeltez igual a 2.0. Además, se utilizaron diez curvas de esfuerzo de tensión-deformación normal. De ambos ensayes no se reportaron propiedades físicas. La instrumentación se realizó con galgas extensométricas, ver Figura 3 (Córdoba, 2014).

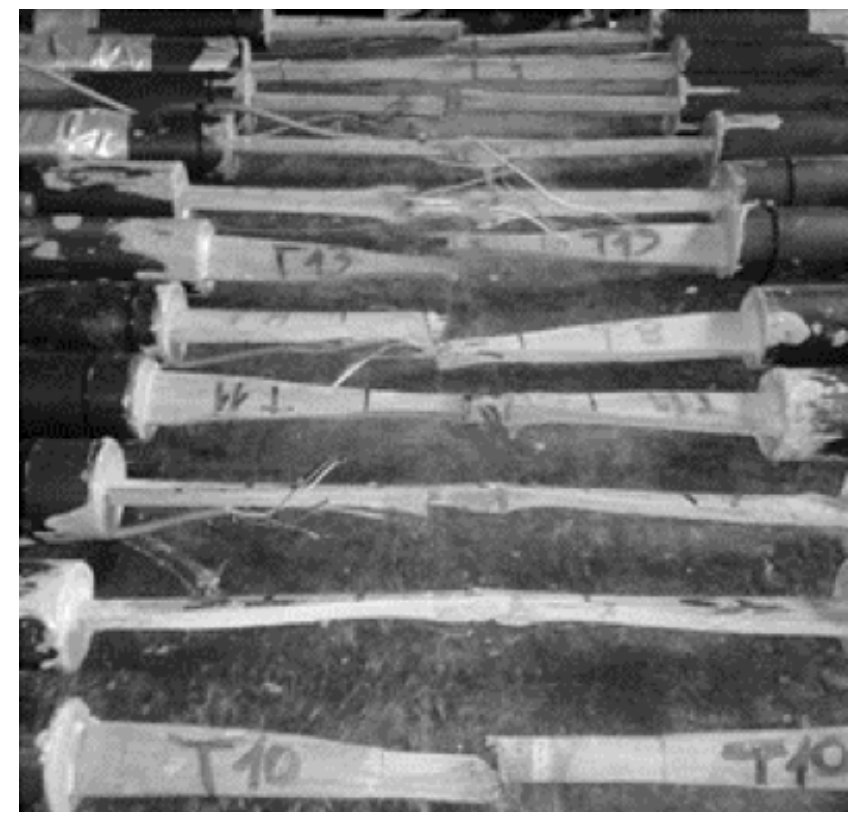

Figura 3.Especímenes en tensión después de la prueba (Córdoba, 2014)

\section{III.3. Datos obtenidos en Colombia}

En Colombia se realizaron estudios con el objeto de evaluar la densidad, contenido de humedad, resistencia a compresión y módulo de elasticidad de la especie Guadua Angustifolia Kunth. Los culmos fueron recolectados en dos departamentos de Colombia (Quindío y Caldas). De este documento se utilizaron 20 curvas reportadas de esfuerzo normal a compresión-deformación 
axial, las cuales fueron obtenidas en especímenes con relación de esbeltez promedio de 2.0 , densidad igual a 0.59 y porcentaje de humedad de $12.76 \%$. Los especímenes fueron instrumentados con galgas extensométricas y la carga fue aplicada con una velocidad de $0.01 \mathrm{~mm} / \mathrm{s}$ (González, 2006).

III.4. Propuesta para la evaluación de la curva media de una serie de curvas experimentales

En la bibliografía no se encontró un procedimiento para el cálculo de la curva media a partir de curvas experimentales. Por tal razón, los autores desarrollaron la propuesta aquí presentada. La Figura 4a presenta una serie de curvas experimentales (curvas 1, 2 y 3 ) sobre las cuales se requiere dibujar líneas de control (cc1 y cc2), con puntos de intersección P1 a P3 en la línea Cc1 y P4 a P6 en la línea cc2. En estas gráficas, las abscisas representan la deformación y las ordenadas representan los esfuerzos. Las coordenadas del punto medio $\mathrm{P}_{\mathrm{i}}\left(\boldsymbol{\varepsilon}_{c c i}, \boldsymbol{\sigma}_{c c i}\right)$ de las tres curvas experimentales intersecadas por la línea CC1 son definidas por las ecuaciones (1) y (2).

$$
\begin{gathered}
\varepsilon_{c c 1}=\left(\varepsilon_{1}+\varepsilon_{2}+\varepsilon_{3}\right) / 3 \\
\sigma_{c c 1}=\left(\sigma_{1}+\sigma_{2}+\sigma_{3}\right) / 3
\end{gathered}
$$

La Figura 4b representa la curva 2 y la línea de control cc1, donde los puntos C22 y C33 pertenecen a la curva experimental y entre éstos se tiene el punto P2, que es la intersección con la curva experimental y coordenadas desconocidas. Considerando que se propone la línea de control cc1, entonces los ángulos $\theta_{1}$ a $\theta_{3}$, así como las coordenadas de C22, c23 y Po son conocidos; por lo cual es posible plantear la ecuación (3). De acuerdo con la Figura 4c se obtiene la ecuación (4).

$$
\begin{aligned}
& V=L \sin \left(\theta_{1}-\theta_{3}\right) / \sin \left(180-\theta_{1}\right) \\
& Z=V \sin \theta_{1} / \sin \left(180-\theta_{1}-\theta_{2}\right)
\end{aligned}
$$

La variación del esfuerzo y la deformación respecto al punto c22 se evalúa mediante las ecuaciones (5) y (6), Figura 4 d. Con las ecuaciones (7) y (8) se definen las coordenadas del punto P2, en forma idéntica se obtienen las coordenadas de los puntos P1 y P3.

$$
\begin{aligned}
& \Delta \varepsilon=Z \cos \theta_{2} \\
& \Delta \sigma=Z \sin \theta_{2} \\
& \varepsilon_{2}=\varepsilon_{C 22}+\Delta \varepsilon \\
& \sigma_{2}=\sigma_{C 22}+\Delta \sigma
\end{aligned}
$$




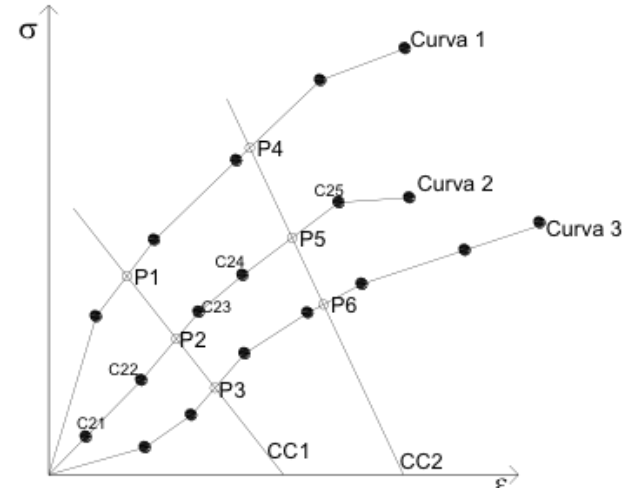

a)

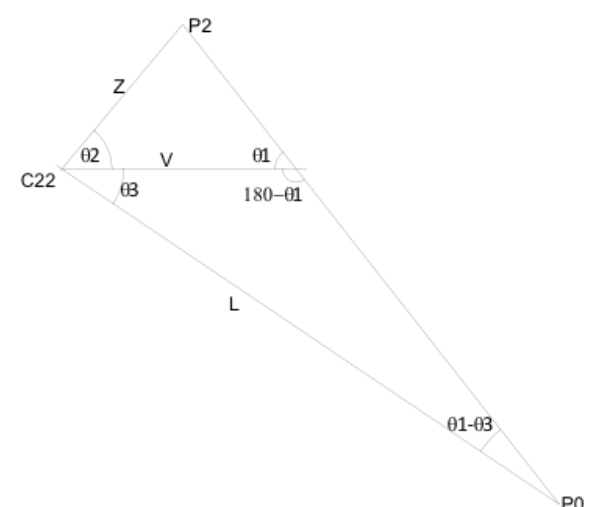

c)

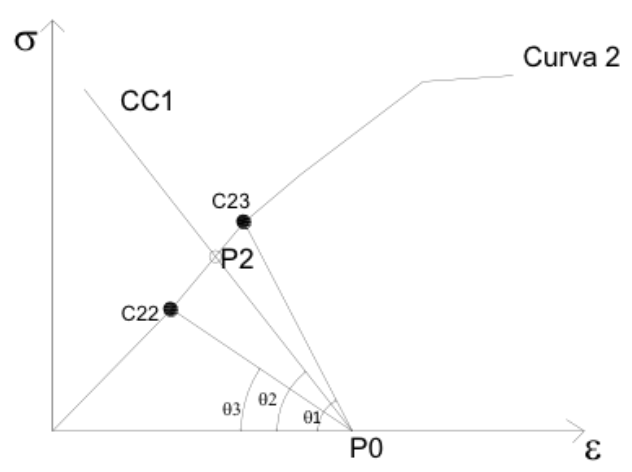

b)

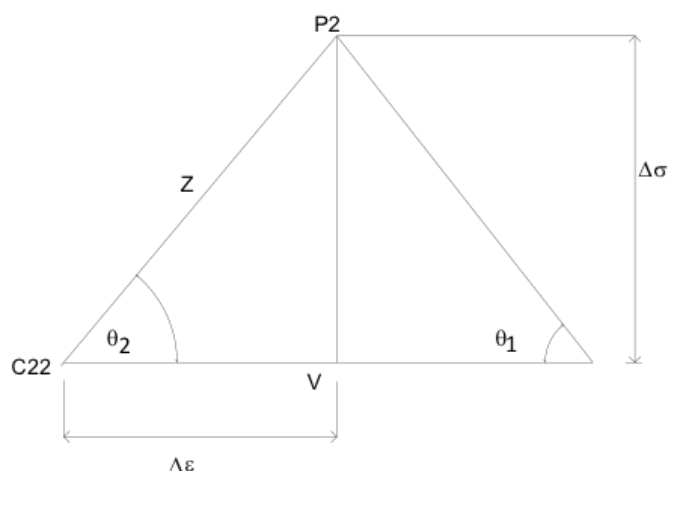

d)

Figura 4. Propuesta para evaluar la curva media de tres curvas experimentales

Finalmente, teniendo las coordenadas de los puntos P1, P2 y P3 y mediante las ecuaciones (1) - (2) se evalúa la coordenada del punto medio de todas las curvas experimentales interceptadas por la línea de control cc1. El procedimiento se repite con la línea de control cc2 (Figura 4a) y así sucesivamente, hasta cubrir la longitud de las curvas experimentales. Este proceso fue automatizado mediante un algoritmo desarrollado en el software libre (Scilab Enterprises, 2014).

\section{RESULTADOS}

\section{IV.1. Ensayes en México}

La Tabla 2 presenta los resultados de contenido de humedad, resistencia y densidad de los especímenes ensayados a compresión. El valor medio del contenido de humedad ( $\mathrm{CH}$ ) fue $9.5 \%$ para la especie Bambusa Oldhamii y $7.8 \%$ en la especie Guadua Angustifolia Kunth. Los valores medios del 
esfuerzo a compresión ( $\sigma_{m c}$ y densidad (D) son: a) 56.1 мpa y 0.66 para Bambusa Oldhamii, b) 49.1 мPa y 0.55 para Guadua Angustifolia Kunth, respectivamente. La última columna de la tabla 2 indica los tres tipos de falla (F) y significan: a) Cortante paralelo (CP), b) Aplastamiento (A), c) Falla combinada de cortante paralelo y aplastamiento (CPA), la Figura 5 presenta cada una de éstas. En la Figura 6 se muestran diez curvas de especímenes ensayados a compresión de Bambusa Oldhamii, en las cuales se midió el módulo de elasticidad medio $\left(E_{m c}\right)$, entre el $20 \%$ y $80 \%$ del esfuerzo máximo, igual a 24.6 GPa con un coeficiente de variación ( $C V)$ de $32 \%$. En la Figura 7 se presentan seis curvas a compresión de la Guadua Angustifolia Kunth donde se registró un valor medio de $E_{m c}=18.4 \mathrm{GPa}$.

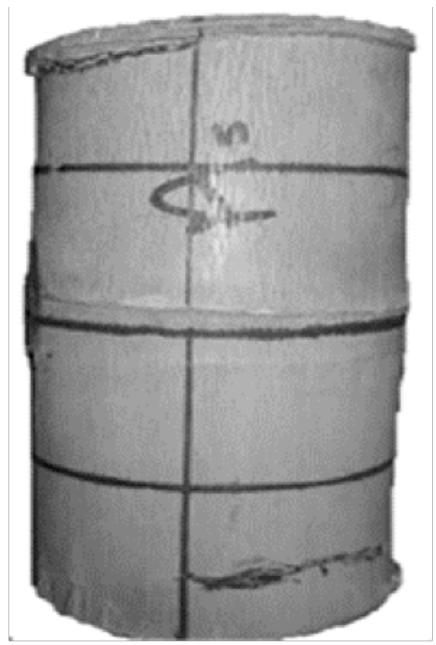

a)

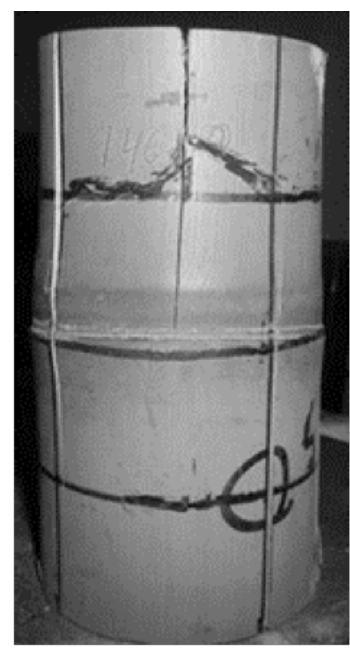

b)

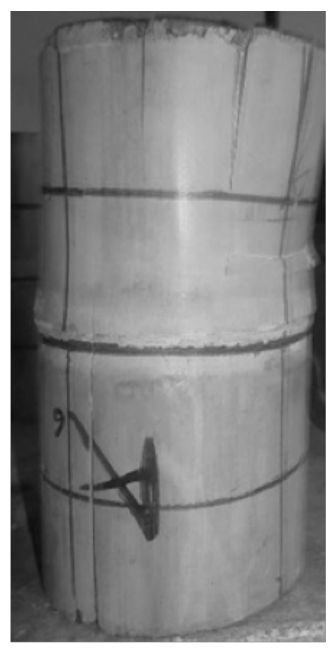

c)

Figura 5. a) Falla por aplastamiento (A) en Guadua Angustifolia Kunth, b) Falla por corte paralelo (CP) en Bambusa Oldhamii, c) Falla por corte paralelo y aplastamiento (CPA) en Guadua Angustifolia Kunth.

\section{Tabla 2}

Resultados de las pruebas de compresión y físicas

\begin{tabular}{|c|c|c|c|c|c|c|}
\hline$\#$ & Con nodo & RE & $\mathrm{CH} \%$ & $\sigma_{m c} \mathrm{MPa}$ & $D$ & Falla \\
\hline 1 & & 1.6 & 16.5 & 50.0 & 0.47 & $\mathrm{CP}$ \\
\hline 2 & Bambusa Oldhamii sin nodo & 2.0 & 8.7 & 53.0 & 0.46 & $\mathrm{CP}$ \\
\hline 3 & & 1.8 & 8.3 & 65.5 & 0.60 & $\mathrm{CP}$ \\
\hline 4 & & 1.9 & 8.8 & 61.0 & 0.80 & $\mathrm{CP}$ \\
\hline 5 & & 2.0 & 9.6 & 61.0 & 0.79 & CPA \\
\hline 6 & & 1.8 & 9.7 & 66.0 & 0.71 & CPA \\
\hline 7 & Bambusa Oldhamii con nodo & 1.9 & 8.9 & 40.0 & 0.64 & $A$ \\
\hline 8 & & 2.0 & 9.9 & 65.5 & 0.84 & $\mathrm{CP}$ \\
\hline 9 & & 1.9 & 9.7 & 59.0 & 0.72 & $A$ \\
\hline 10 & & 2.0 & 4.6 & 40.5 & 0.58 & CPA \\
\hline
\end{tabular}




\begin{tabular}{|c|c|c|c|c|c|c|}
\hline 11 & \multirow{6}{*}{ Guadua Angustifolia Kunth } & 1.9 & 8.9 & 53.0 & 0.31 & A \\
\hline 12 & & 1.9 & 8.4 & 41.0 & 0.64 & CPA \\
\hline 13 & & 1.9 & 8.0 & 68.0 & 0.84 & $\mathrm{CP}$ \\
\hline 14 & & 1.9 & 8.3 & 45.5 & 0.51 & A \\
\hline 15 & & 1.8 & 8.8 & 46.0 & 0.52 & A \\
\hline 16 & & 1.8 & 4.1 & 51.0 & 0.51 & $\mathrm{CP}$ \\
\hline
\end{tabular}

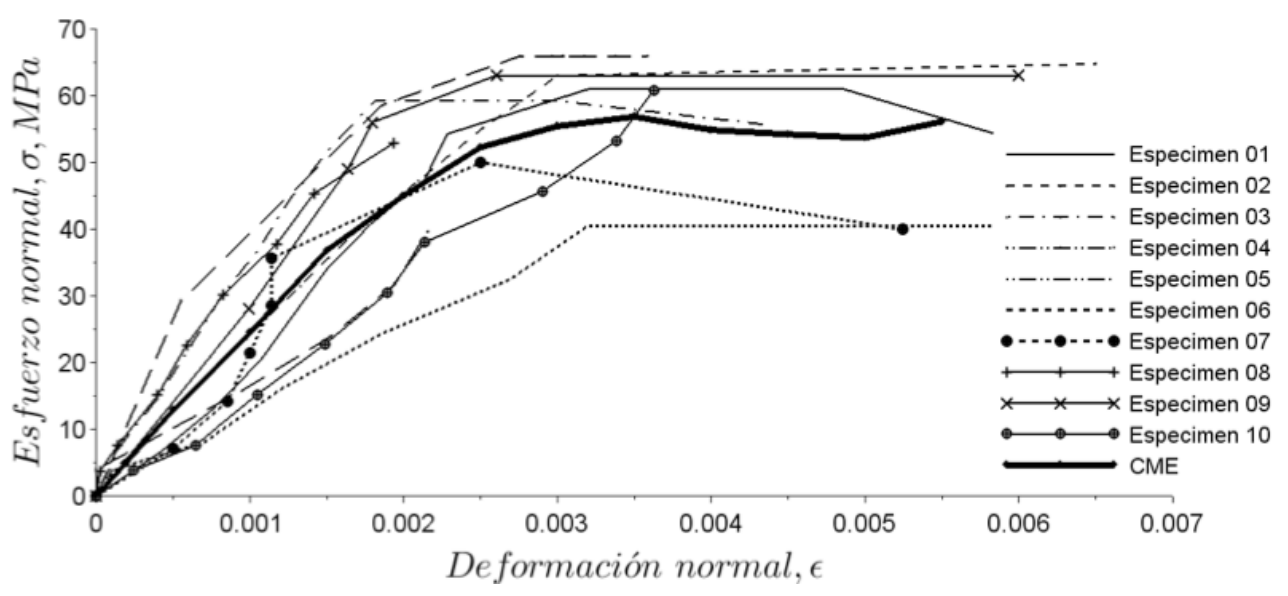

Figura 6. Curvas de esfuerzo normal-deformación normal (Bambusa Oldhamii, México)

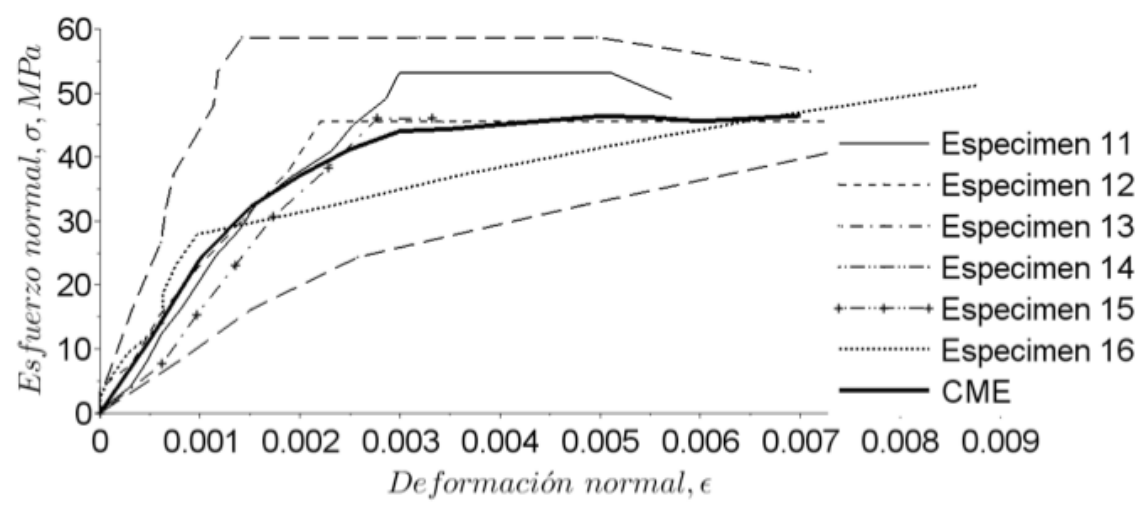

Figura 7. Curvas de esfuerzo normal-deformación normal (Guadua Angustifolia Kunth México)

Con la idea de representar el comportamiento mecánico ante carga axial, se obtuvo la curva media experimental (CME) de las diez curvas experimentales de Bambusa Oldhamii, Figura 6, el cálculo se hizo a cada $\Delta \boldsymbol{\varepsilon}=0.0005$, desde $\varepsilon=0.0005$ hasta $\varepsilon=0.0055$, de acuerdo con el proceso definido en II.4. La última línea de control interseca al menos cuatro curvas experimentales. A continuación, mediante análisis de mínimos cuadrados se obtuvo la ecuación (9), que define la curva media propuesta (CMP), donde se distinguen dos partes, si la deformación $\boldsymbol{\varepsilon} \leq 0.0035$ se establece una ecuación de segundo grado, en caso contrario se tiene una ecuación de primer grado. Este proceso 
se repite para los datos de Guadua Angustifolia Kunth, ver Figura 7 y ecuación (10), para la cual la deformación máxima propuesta fue 0.007 .

$$
\begin{aligned}
& \sigma=\left\{\begin{array}{c}
-3797030.80 \varepsilon^{2}+29819.41 \varepsilon \text { si } 0 \leq \varepsilon \leq 0.0035 \\
-864.92 \varepsilon+54.83 \text { si } 0.0035<\varepsilon \leq 0.0055
\end{array}\right. \\
& \sigma=\left\{\begin{array}{c}
-4322731.30 \varepsilon^{2}+27500.32 \varepsilon \text { si } \leq 0.0 \varepsilon \leq 0.0030 \\
601.79 \varepsilon+42.56 \text { si } 0.0030<\varepsilon \leq 0.007
\end{array}\right.
\end{aligned}
$$

Respecto a la prueba de tensión, la Figura 8 contiene nueve curvas experimentales de la especie Bambusa Oldhamii, también se presenta la curva media experimental ( $\mathrm{CME}$ ). El valor medio del módulo elástico, medido al $50 \%$ del esfuerzo máximo fue $E_{m t}=14.92 \mathrm{GPa}$ con un $\mathrm{CV}=39 \%$. El valor medio del esfuerzo máximo de las nueve muestras alcanzó $\sigma_{m t}=273.30$ MPa y $C V=14.4 \%$, el cual estuvo asociado a una deformación $\varepsilon=0.0197$. La ecuación (11) representa la CMP del esfuerzo normal-deformación normal, en este caso $\varepsilon_{1}=0.01$. La Figura 9 presenta el estado final de un espécimen después del ensaye.

$$
\sigma=\left\{\begin{array}{c}
14553.86 \varepsilon \text { si } 0.0 \varepsilon \leq 0.010 \\
8111.07\left(\varepsilon-\varepsilon_{1}\right)+145.54 \text { si } 0.010<\varepsilon \leq 0.024
\end{array}\right.
$$

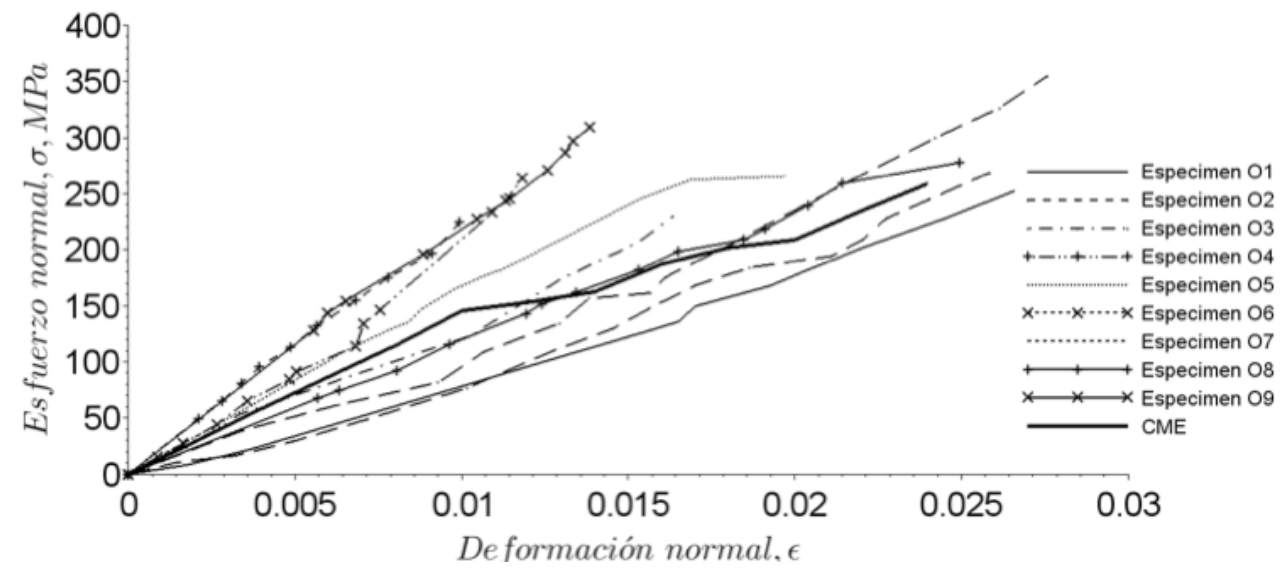

Figura 8. Curvas de esfuerzo normal de tensión-deformación normal para Bambusa Oldhamii México (Mendoza, 2014)

IV.2. Curvas medias experimentales y propuestas para la especie Guadua Angustifolia Kunth de Ecuador y Colombia

De acuerdo con la metodología presentada, se obtuvieron las curvas medias experimentales (CME) y propuestas (CMP) del esfuerzo a compresióndeformación normal. En el caso de Ecuador, la ecuación (12) representa la CMP a partir de la curva media experimental y que es válida para defor- 
maciones $\boldsymbol{\varepsilon} \leq$ 0.0035. En los ensayes de Colombia se propuso la ecuación (13) de tipo parabólica hasta $\boldsymbol{\varepsilon} \leq 0.0040$ y lineal de este punto hasta $\boldsymbol{\varepsilon}=0.0070$, ver Figuras 10 y 11.

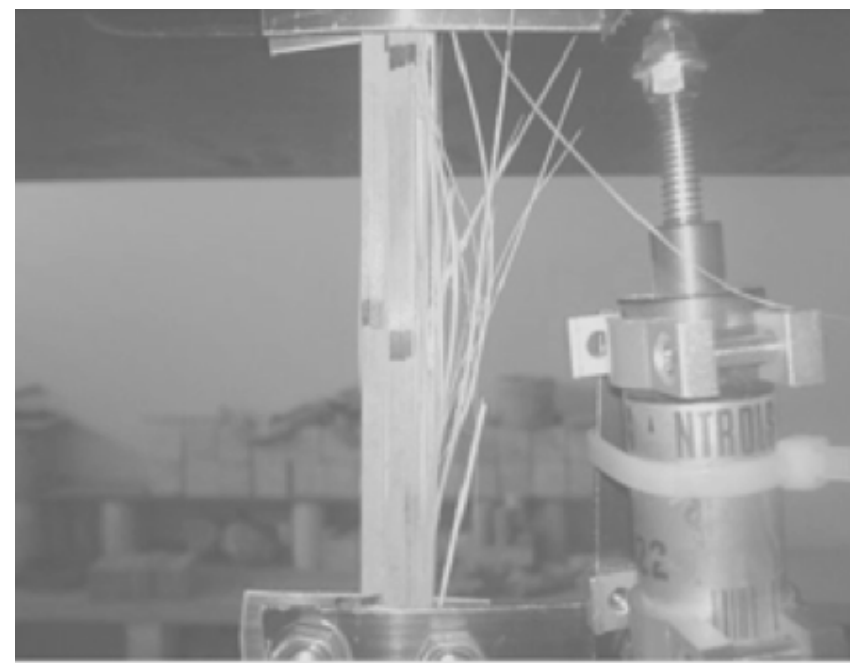

Figura 9. Falla de un espécimen a tensión (Mendoza, 2014)

El valor medio del esfuerzo a compresión en Ecuador fue $\sigma_{m c}=48.9 \mathrm{MPa}$ y $C V=10.7 \%$, mientras que el módulo elástico a compresión registró $E_{m c}=25.5 \mathrm{GPa}$ con $C V=21 \%$. En el caso de Colombia se registró un valor medio de esfuerzo a compresión $\sigma_{m c}=52.31 \mathrm{MPa}$ con $C V=16.2 \%$, módulo de elasticidad $E_{m c}=17.8$ GPa y $C V=42 \%$.

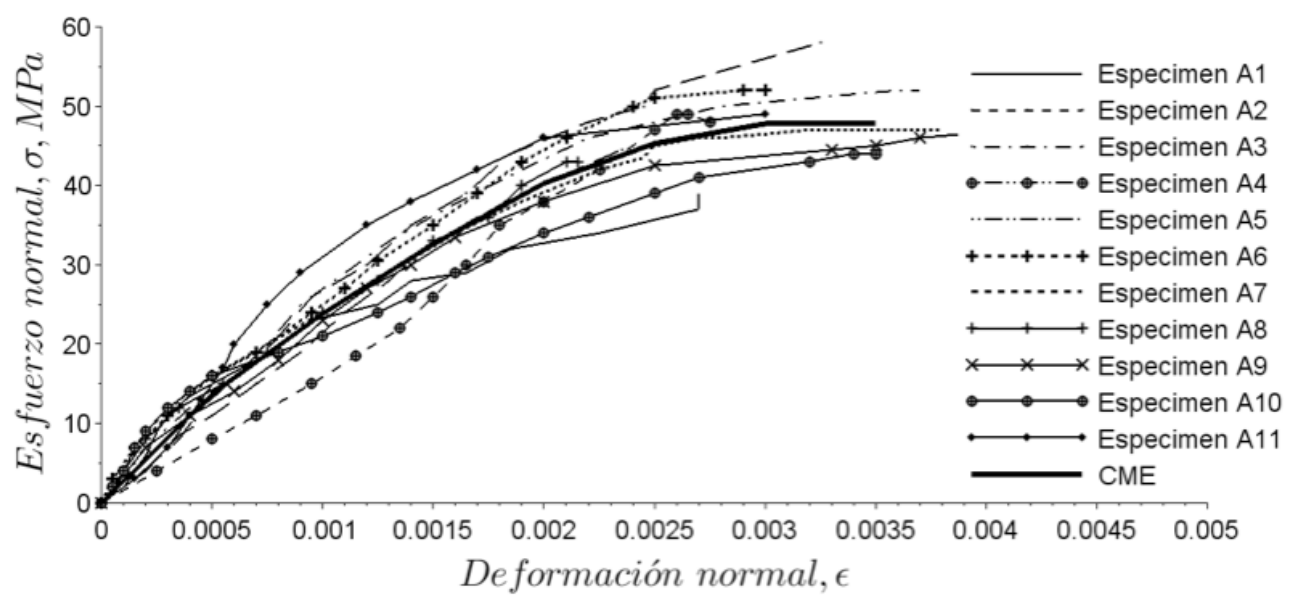

Figura 10. Curvas esfuerzo normal-deformación normal de Guadua Angustifolia Kunth, Ecuador (Córdoba, 2014)

$\sigma=-4186304.80 \varepsilon^{2}+28423.46 \varepsilon$ si $0 \leq \varepsilon \leq 0.0035$ 


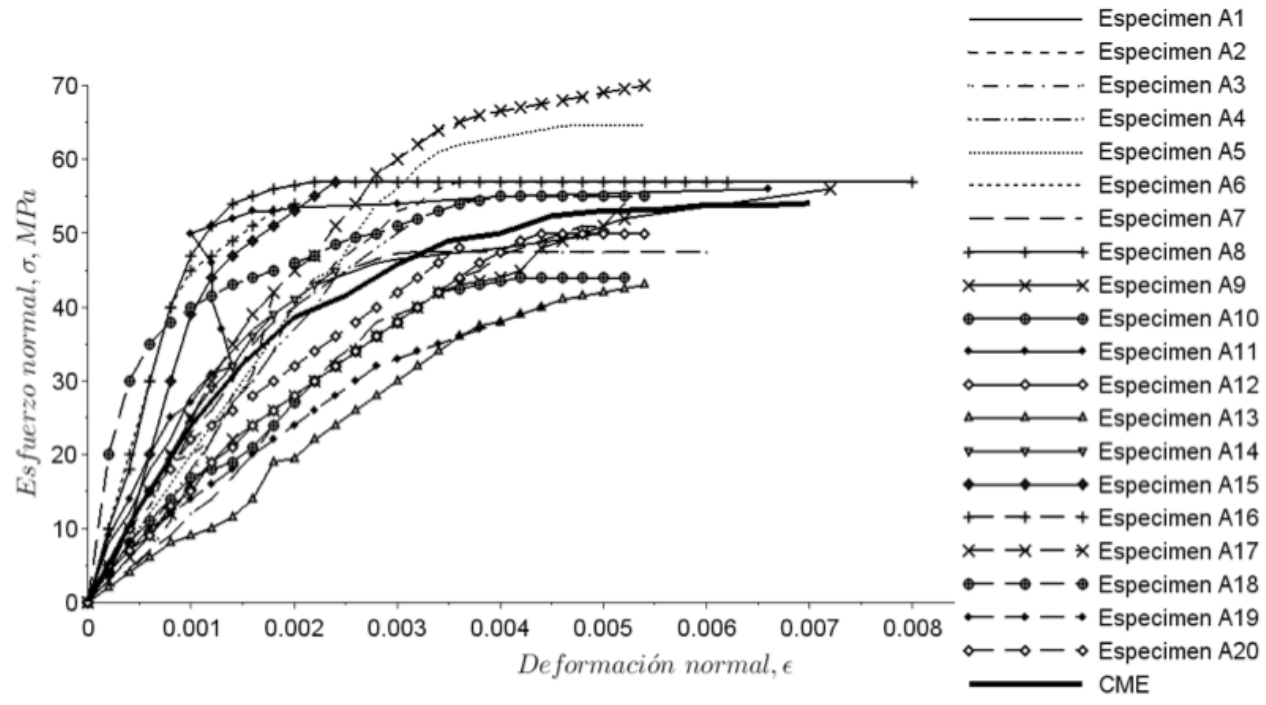

Figura 11. Curvas de esfuerzo normal-deformación normal de Angustifolia Colombia (González, 2006)

$$
\sigma=\left\{\begin{array}{c}
-2817267 \varepsilon^{2}+24243.87 \varepsilon \text { si } 0 \leq \varepsilon \leq 0.0040 \\
721.42 \varepsilon+49.01 \text { si } 0.0040<\varepsilon \leq 0.007
\end{array}\right.
$$

La Figura 12 presenta las gráficas de tensión de diez pruebas realizadas en Ecuador, así como la curva media experimental. La CMP es definida con la ecuación (14), para este caso $\boldsymbol{\varepsilon}_{1}=0.007$. El valor medio del esfuerzo a tensión fue $\sigma_{m t}=136.0$ MPa y $C V=7.5 \%$. El módulo elástico resultó $E_{m t}=18.4 \mathrm{GPa}$ con un coeficiente de variación $C V=26 \%$.

$\sigma=\left\{\begin{array}{c}16120.18 \varepsilon \text { si } 0 \leq \varepsilon \leq 0.007 \\ 8177.15\left(\varepsilon-\varepsilon_{1}\right)+112.84 \text { si } 0.0070<\varepsilon \leq 0.009\end{array}\right.$

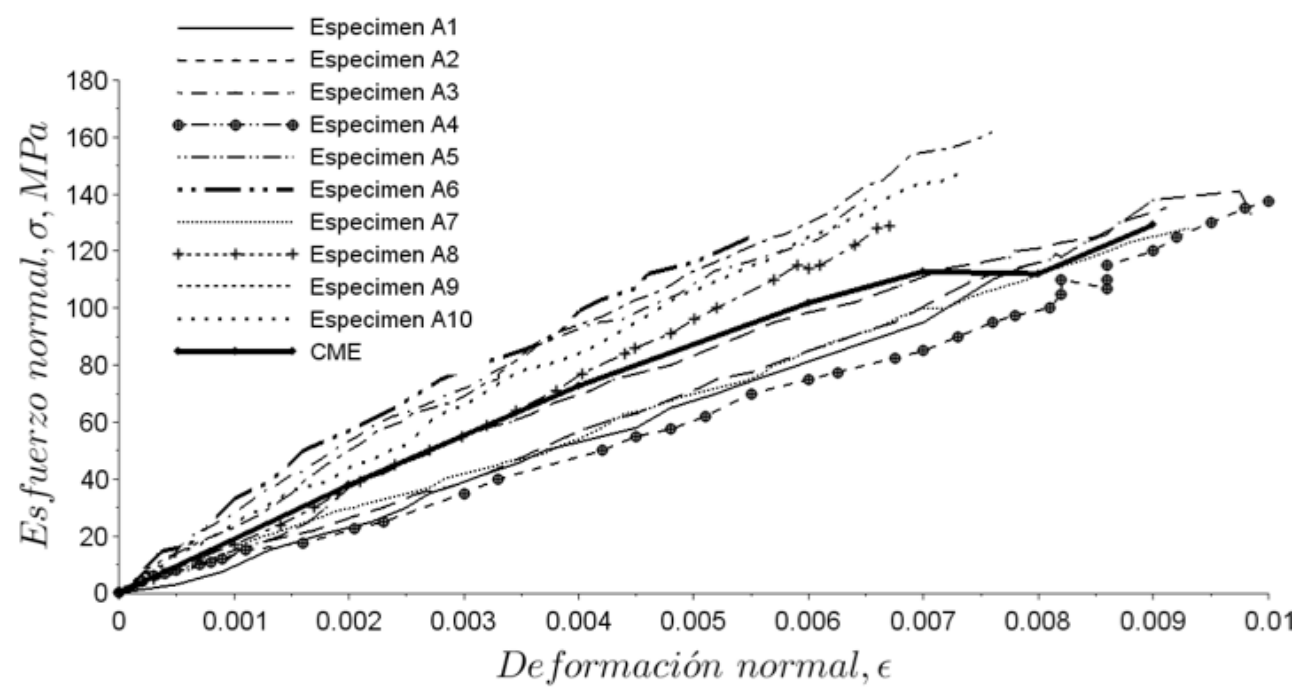

Figura 12. Curvas de esfuerzo normal de tensión-deformación normal de Guadua Angustifolia Ecuador (Córdoba, 2014) 
En la Figura 13 se presentan las curvas medias experimentales (CME) y propuestas (CEP) de compresión y tensión contra la deformación, mientras que la Figura 14 presenta las curvas propuestas completas para Bambusa Oldhamii (México) y Guadua Angustifolia Kunth (Ecuador). La parte negativa es tensión definida por las ecuaciones (11) y (14), la parte positiva representa compresión y se obtiene al graficar las ecuaciones (9) y (12). Para realizar una comparación, se agregó una curva de concreto simple obtenida en la prueba de compresión (Sánchez et al., 2011)

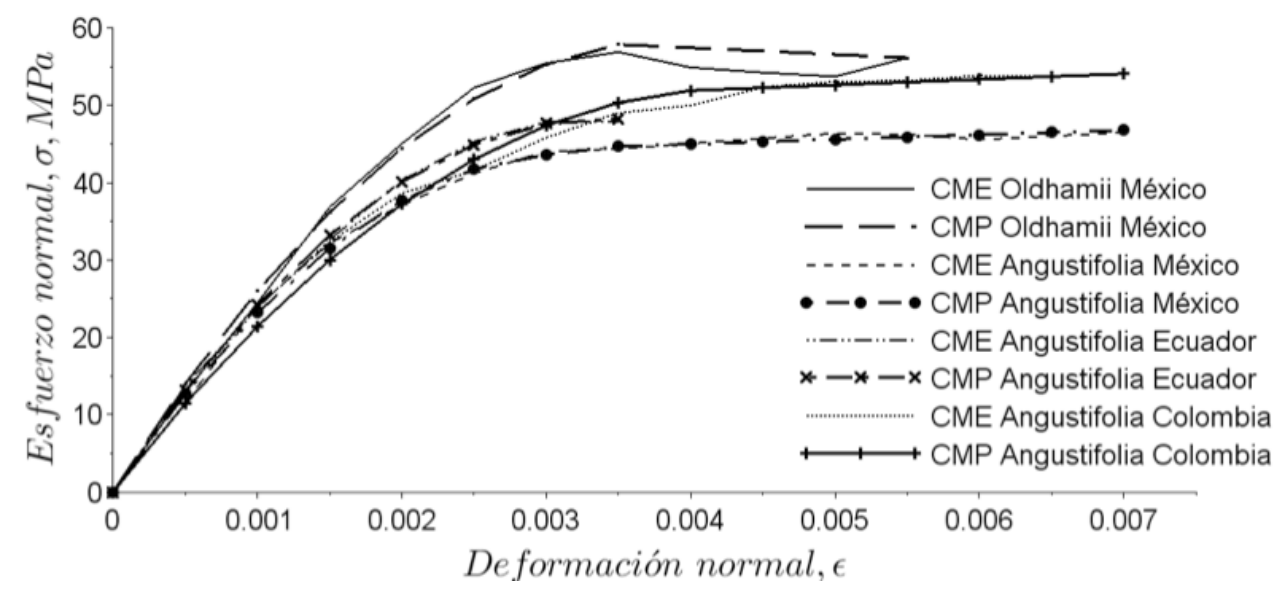

a) Compresión

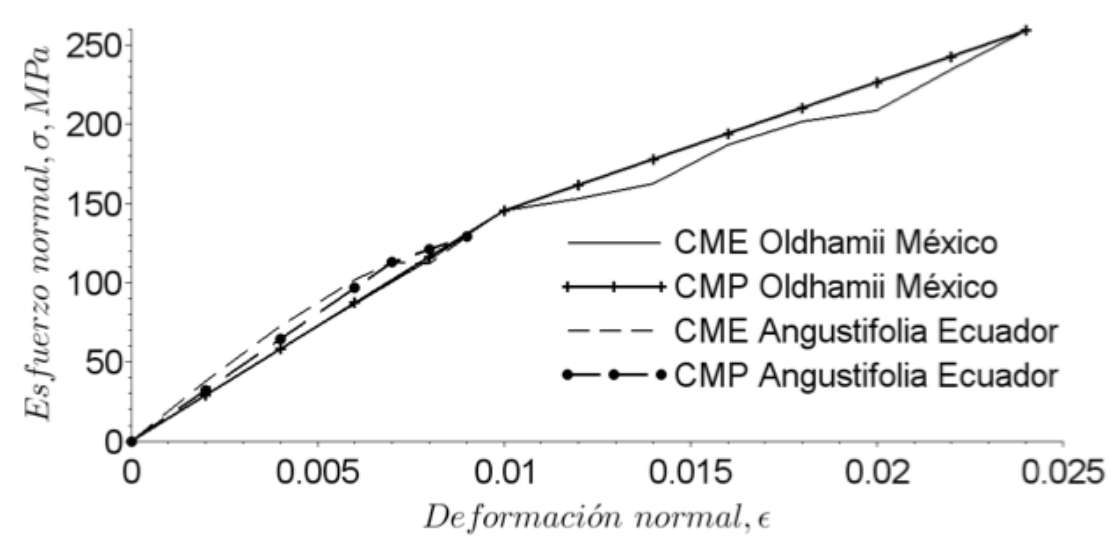

b) Tensión

Figura 13. Comparación de curvas experimentales y propuestas

\section{DISCUSIÓN}

Las Tablas 3 y 4 presentan el resumen de las propiedades mecánicas y físicas de los especímenes ensayados. Respecto a la densidad (D), la Guadua Angustifolia Kunth registró valores semejantes para los casos de México y 
Colombia. Referente al contenido de humedad $(\mathrm{CH})$, los especímenes ensayados en México estaban más secos (7.8\% contra $12.7 \%)$. Respecto a la resistencia a compresión $\left(\boldsymbol{\sigma}_{m c}\right)$, la especie Bambusa Oldamii tiene el mayor valor con 56.1 MPa y la resistencia a compresión de la Guadua Angustifolia Kunth es parecida en los tres estudios; el módulo elástico en compresión de Guadua Angustifolia Kunth $\left(E_{m c}\right)$ obtenido en Ecuador resultó más alto, con un valor menor de la deformación $\left(\boldsymbol{\varepsilon}_{m c}=0.0035\right)$ asociado al esfuerzo medio; los valores de deformación asociados a los esfuerzos medios obtenidos en México y Colombia son similares.

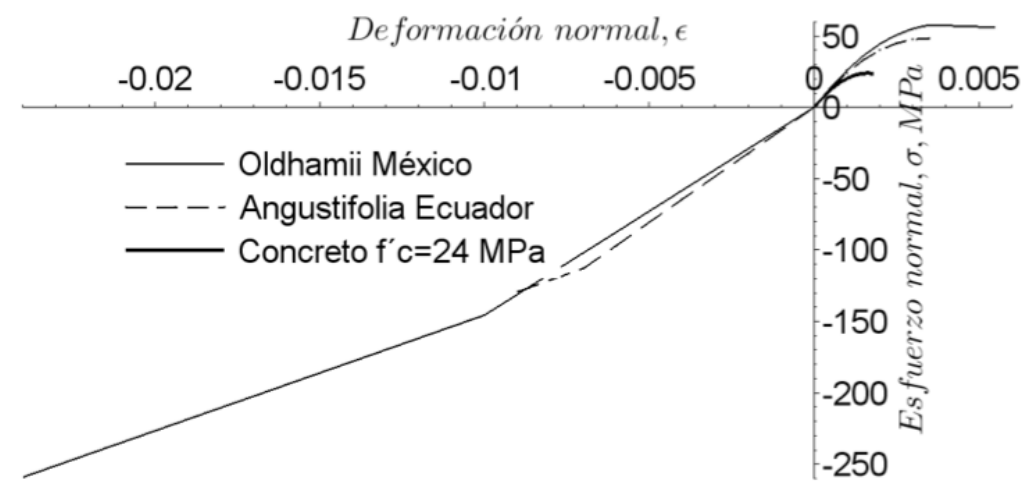

Figura 14. Curvas de comportamiento propuestas ante carga monótona en tensión-compresión

En las pruebas de tensión, nuevamente la Bambusa Oldhamii-México registró el mayor esfuerzo $\left(\sigma_{m t}=273.3 \mathrm{MPa}\right)$ mientras que la Guadua Angustifolia Kunth-Ecuador sólo alcanzó el 49\% de este valor (136.0 MPa). En el caso del módulo elástico $\left(E_{m t}\right)$ se invirtió la situación, la Bambusa Oldhamii-México alcanzó el 81 \% (14.92 GPa) del valor registrado por la Guadua Angustifolia Kunth-Ecuador.

\section{Tabla 3}

Valores medios de propiedades físicas

\begin{tabular}{ccc}
\hline Especie & \multicolumn{2}{c}{ Propiedades físicas } \\
Origen & CH (\%) & $D$ \\
Bambusa Oldhamii/México & 9.5 & 0.66 \\
Guadua Angustifolia Kunth/México & 7.8 & 0.55 \\
Guadua Angustifolia Kunth/Colombia & 12.7 & 0.59 \\
\hline
\end{tabular}


Tabla 4

Valores medios de propiedades mecánicas

\begin{tabular}{cccccccc}
\hline & \multicolumn{5}{c}{ Propiedades mecánicas } \\
Especie & \multicolumn{3}{c}{ Compresión } & \multicolumn{3}{c}{ Tensión } \\
Origen & $\boldsymbol{\sigma}_{m c}(\mathrm{MPa})$ & $E_{m c}(\mathrm{GPa})$ & $\boldsymbol{\varepsilon}_{m c}$ & $\boldsymbol{\sigma}_{m t}(\mathrm{MPa})$ & $E_{m t}(\mathrm{GPa})$ & $\boldsymbol{\varepsilon}_{m t}$ \\
Bambusa Oldhamii/México & 56.1 & 24.6 & 0.0055 & 273.3 & 14.9 & 0.0197 \\
Guadua Angustifolia Kunth/México & 12.7 & 0.59 & & & & \\
Guadua Angustifolia Kunth/Ecuador & 48.9 & 25.5 & 0.0035 & 136.0 & 18.4 & 0.008 \\
Guadua Angustifolia Kunth/Colombia & 52.3 & 17.8 & 0.0057 & & & \\
\hline
\end{tabular}

En las Tablas 3 y 4 se tiene: $C H$, contenido de humedad; $D$, densidad; $\boldsymbol{\sigma}_{m c}$, esfuerzo medio de compresión; $E_{m c}$, módulo de elasticidad a compresión; $\boldsymbol{\varepsilon}_{m c}$, deformación asociada al esfuerzo medio de compresión; $\sigma_{m t}$, esfuerzo medio de tensión; $E_{m t}$, módulo de elasticidad a tensión; $\boldsymbol{\varepsilon}_{m t}$, deformación asociada al esfuerzo medio de tensión.

Referente al modo de falla en las pruebas a compresión no hay un patrón definido, excepto para los cuatro especímenes con nodo de Bambusa Oldhamii que fallaron por cortante paralelo (СP), donde se observó un efecto negativo en la resistencia a compresión.

Las curvas medias propuestas a compresión (CMP) son, en la mayoría de los casos, una combinación de parábolas y rectas, la parte parabólica termina con deformaciones entre 0.0030 y 0.004 , después existe un comportamiento lineal con disminución de rigidez. La Figura 13a muestra un comportamiento similar de la especie Guadua Angustifolia Kunth en los tres países, sin importar los parámetros de ensaye, la geometría y/o las características físicas de los especímenes, ni tampoco la posición que ocupaba el espécimen en la caña de bambú. Así, la pendiente inicial en las tres curvas es semejante hasta deformaciones entre 0.0035 y 0.004, después el material se plastifica.

Con respecto a las pruebas de tensión, la curva media propuesta (CMP) de Bambusa Oldhamii muestra una degradación de rigidez a partir de $\boldsymbol{\varepsilon}=$ 0.01, mientras que en la Guadua Angustifolia Kunth se inicia con $\boldsymbol{\varepsilon}=0.007$. En el mismo orden, la razón de esfuerzos últimos de tensión-compresión experimentales es 4.87 y 2.78 , respectivamente, con una deformación última mayor en la especie Bambusa Oldhamii.

En la Figura 14 se muestran las curvas propuestas de ambas especies en tensión y compresión registradas en México y Ecuador bajo carga monótona, la cual indica la ventaja estructural del bambú respecto al concreto simple. Para este caso, la máxima resistencia del concreto es 20 MPa mientras la especie Bambusa Oldhamii alcanza valores cercanos a 60 MPa, con relaciones similares de esbeltez en los ensayes de ambos materiales (dos para el concreto y 1.9 en el espécimen de bambú). En el lado de tensión el concreto 
no supera esfuerzos mayores al 10\% del esfuerzo en compresión (2.0 MPa), mientras la Bambusa Oldhamii alcanza hasta 250 мра.

\section{CONCLUSIONES}

Las curvas medias experimentales de esfuerzo a compresión normaldeformación normal de la Guadua Angustifolia Kunth obtenidas en México, Ecuador y Colombia tienen la misma forma.

Los resultados en especímenes de bambú y concreto simple, con idénticas relaciones de esbeltez, muestran una mayor ductilidad y resistencia del primero respecto al segundo, la resistencia máxima a compresión de 60 MPa registrada en la Bambusa Oldhamii correspondería a un concreto de alta resistencia.

En ambas variedades de bambú, la curva de esfuerzo de tensióndeformación normal presenta un comportamiento elastoplástico. En la especie Bambusa Oldhamii la pendiente del tramo plástico es 55\% de la pendiente del tramo elástico, la razón se reduce a 51\% en la especie Guadua Angustifolia Kunth.

La semejanza de las curvas medias en ensayes a compresión de la Guadua Angustifolia Kunth obtenidas en los tres países, sin importar el procedimiento de prueba ni las características y origen de los especímenes, indica la posible caracterización del comportamiento del material, similar al realizado para el concreto simple o el acero estructural. Así, las curvas compresión-tensión-deformación axial obtenidas pueden ser utilizadas en el análisis de estructuras elaboradas con este material.

Bajo ciertas condiciones, el bambú tiene un mejor comportamiento respecto al registrado en el concreto simple. Sin embargo, deben revisarse las desventajas del material, específicamente la durabilidad y resistencia al fuego, que pueden reducir el período de vida útil de las estructuras construidas con este material. Otra problemática en la fase de diseño es el desconocimiento a priori de las propiedades geométricas de los culmos, a diferencia de los perfiles de acero, elementos de concreto o madera donde se conoce con exactitud las secciones transversales.

Finalmente, este biomaterial sería una opción de construcción en la región de la República Mexicana formada por Guerrero, Oaxaca y Chiapas, donde existen las condiciones meteorológicas apropiadas, ayudaría a reducir la falta de vivienda, disminuir el riesgo de la sociedad ante los efectos de fenómenos naturales y reducir los costos de la construcción. 


\section{REFERENCIAS}

Álvarez, C. (2012). Comportamiento mecánico de las conexiones en los elementos de bambú para estructuras ligeras: El caso de las especies del trópico de Veracruz. (Tesis de doctorado). Universidad Politécnica de Madrid, Madrid. Obtenido de http://oa.upm.es/14530/1/03_2012_ ENRIQUE_ROBERTO_ALVAREZ_CASTILLA.pdf

Ascencio, L. O. (2010). El uso del bambú como alternativa para viviendas de interés social. México: Gráficas del Sur.

Barragán-Trinidad, R., Cuevas, A., Sánchez, M., \& Ortega, R. (2014). Ensaye de sistemas de cubiertas y marcos planos de bambú como una alternativa sustentable en la construcción de vivienda económica. Foro de estudios sobre Guerrero, 1(1), 209-213. Obtenido de http://www.fesgro.mx/journal/ Extenso/COCYTIEG\%20\%2OJOURNAL\%20I.pdf

Camacho, V., \& Páez, I. (2002). Estudio de conexiones en guadua, solicitadas a momento flector. (Tesis de Ingeniería Civil). Universidad Nacional de Colombia, Bogotá. Obtenido de http://www.sigguadua.gov.co/sites/ default/files/archivos/o3.pdf

Córdova, P. (2014). Obtención de las propiedades mecánicas y estructurales de la caña guadúa angustifolia kunth del ecuador (Tesis de Ingeniería Civil). Universidad Católica de Santiago de Guayaquil, Ecuador. Obtenido de http://repositorio.ucsg.edu.ec/handle/3317/1423

Dixon, P., \& Gibson. (2014). The structure and mechanics of Moso bamboo material. J.R: Soc. Interface 11, 1-12. https://doi.org/10.1098/rsif.2014.0321

Gobierno de la Ciudad de México. (2017). Normas técnicas complementarias de diseño y construcción de estructuras de concreto. CDMx. Obtenido de https://www.smig.org.mx/archivos/NTc2017/normas-tecnicas-complementarias-reglamento-construcciones-cdmx-2017.pdf

González, C. O., \& Robles, F. F. (2005). Aspectos fundamentales del concreto reforzado (4ta ed.). México: Limusa.

González, C. E. (2006). Resistencia a la compresión paralela a la fibra de la guadua angustifolia y determinación del módulo de elasticidad. (Tesis de Ingeniería Civil). Universidad Nacional de Colombia, Bogotá. Obtenido de https://www.usmp.edu.pe/centro_bambu_peru/pdf/Resistencia_ compresion_paralela_fibra_guadua_determinacion_modulo_elastic.pdf

Instituto Mexicano de la Construcción en Acero. (2014). Manual de construcción en acero (5th ed.). México: Limusa.

International Organization for Standardization. (2004). BambooDetermination of physical and mechanical properties, Parte 1, Requirements. ISO 22157-1:2004. Switzerland.

Kakkad, M. D., \& Sanghvi, C. (2011). Comparative study of bamboo (ikra) housing system with modern construction practices. Paper presented at 
the National Conference on Recent Trends in Engineering \& Technology, BVM Engineering College, Nagar, Gujarat, India. Retrieved from http:// www.bvmengineering.ac.in/misc/docs/published-2opapers/civilstruct/ struct/101078.pdf

Mendoza, J. N. (2014). Propiedades físicas y mecánicas del bambú: Perspectivas de empleo para la construcción de viviendas económicas. (Tesis de Ingeniería Civil). Universidad Autónoma de Guerrero, México.

Ordóñez- Candelaria, V. R., \& Bárcenas-Pazos, G. M. (2014). Propiedades físicas y mecánicas de tres especies de guaduas mexicanas (guadua aculeata, guadua amplexifolia y guadua velutina). Maderas y Bosques, 20(2), 111-125. Obtenido de http://www.scielo.org.mx/scielo.php?script=sci_arttext\&pi $\mathrm{d}=\mathrm{S} 1405-04712014000200010$

Sánchez, S., Barragán, R., García, V. M., Delgado, D., \& Pastrana, E. I. (2011). Experimental and numerical study of confined masonry walls subject to lateral loads. México: Ediciones e Impresiones.

Sánchez, S., Arroyo, R., \& Jerez, S. (2010). Modelo de un grado de libertad para evaluar la curva carga lateral-distorsión en muros de mampostería confinada. Ingeniería Sísmica, 83, 25-42. Obtenido de http://www.scielo. org.mx/scielo.php?pid=S0185-092X2010000200002\&script=sci_arttext

Sánchez, M., Espuma, J., \& Roux, R. (2016). El bambú como elemento estructural: la especie Guadua Amplexifolia. Nova Scientia, 8(17), 657-677. Obtenido dehttp://www.redalyc.org/articulo.oa?id=203349086032.

Scilab Enterprises. (2014). Scilab 5.4.1 (Cecill License; ed.). Retrieve from https://www.scilab.org/download/6.0.2

Sreemathi, I. (2002). Guidelines for building bamboo-reinforced masonry in earthquake-prone areas in India. (Master Thesis). University of Southern California, California. Retrieved from https://www.sheltercluster.org/ sites/default/files/docs/Guidelines_for_building_bamboo-reinforced_ masonry_in_earthquake_zones.pdf 


\section{LISTA DE ACRÓNIMOS Y SÍMBOLOS}

A, $\quad$ Falla por aplastamiento

CH, Contenido de humedad en \%

CME, Curva media experimental

смP, Curva media propuesta

$C P, \quad$ Falla por cortante paralelo

CPA, Falla por cortante paralelo y aplastamiento

$C V, \quad$ Coeficiente de variación

$D$, Densidad adimensional

$E_{m c}$, Módulo de elasticidad medio a compresión, GPa

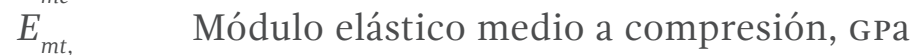

LDVT, Transductor de desplazamiento lineal

RE, Relación de esbeltez

$\boldsymbol{\varepsilon}, \quad$ Deformación normal adimesional

$\boldsymbol{\varepsilon}_{1}$, Deformación normal elástica a tensión

$\boldsymbol{\sigma}, \quad$ Esfuerzo normal

$\boldsymbol{\sigma}_{m c}$ Esfuerzo medio a compresión, мPa

$\boldsymbol{\sigma}_{m t}, \quad$ Esfuerzo medio a tensión, мpa 